http://jmscr.igmpublication.org/home/ ISSN (e)-2347-176x ISSN (p) 2455-0450

crossref DOI: https://dx.doi.org/10.18535/jmscr/v9i5.29

\title{
Effects of Lockdown and Movement Restriction on Glycaemic Status among Diabetics ELMoGS Study
}

\author{
Authors \\ Swastik Sharma*1, Dr Harharpreet Kaur ${ }^{2}$ \\ ${ }^{1}$ MBBS Intern, Maharishi Markandeshwar Medical College and Hospital, Solan, Himachal Pradesh \\ 173229, India \\ ${ }^{2}$ M.D., Head of Department, Department of Medicine, Maharishi Markandeshwar Medical College and \\ Hospital, Solan, H.P. 173229 \\ Corresponding Author \\ Swastik Sharma
}

\begin{abstract}
Background and Objectives: Widespread, lockdowns were placed throughout the world due to the risk of COVID infection. This has caused, most of the population including those with chronic diseases to be restricted to their homes with the possibility of reduced physical activity and weight gain. The patients of diabetes mellitus are more prone to incur COVID 19 infection. The aim of the study was to determine the effect of this change in life style on their disease status. It will also help prepare the target population for possibilities of any future lockdowns.

Material \& Methods: The study was conducted during the National Lockdown(s) with the help of voluntary collection of data regarding the glycaemic status, self-monitored by the participants at home. A questionnaire ${ }^{1}$ designed to filter out biases and match the relevance of symptomatology was employed. Two groups were formed, one with digital monitoring of blood sugar and other with empirical symptomatology reporting. Data was collected over a period of 60 days during the first week of April to last week of May, 2020.

Results: Fifty five percent respondents were found to have significantly high blood glucose compared to before the lockdown, and nineteen percent had symptoms consistent with hyperglycaemia. In total 74 percent of the surveyed were hyperglycaemic.

Interpretation and Conclusions: The survey studies have indicated that many patients of diabetes mellitus can be expected to have a deterioration in the glycaemic status and therefore worsened signs, symptoms and more complications of this disease. Clinicians will hence find it in good stride to correlate these complaints with the effects of lockdown.
\end{abstract}

Keywords: COVID, Diabetes, Hyperglycaemia, Lifestyle, Lockdown, SARS-CoV-2, Public Health.

\section{Introduction}

Glycaemic control in chronic diabetics is primarily dependent on drug-based management and to a major extent on the level of physical activity that the person is involved in. This, in effect means that the dosage of drugs (including extrinsic insulin) and the lifestyle of patients affect glycaemic status. Change in these factors should affect the levels of blood glucose. This study is an attempt to find out the extent to which 
the above factors affect the glycaemic control in the diabetic patients during lockdown.

The lockdown in India was imposed on 24 March and extended up to May 31 2020.All this meant that the persons suffering from Diabetes Mellitus had lesser physical activity (which may include the travel to and from the workplace and within it, daily home supply runs etc.). It reduced, to such levels that blood sugar levels may show variations beyond what the person's body dynamics and the drug intake/ dosages might have been able to control so far. Also, it is unlikely that many persons have significantly or at all, changed their daily food intake in view of these facts. This aspect is also discussed further.

The objective, further, is to help prepare the at risk population for possibilities of any future lockdowns by means of dietary advice and adding exercise to the daily routine. It is also important for clinicians to know if any need for special care vis-a-vis the diabetic medication is needed in such a scenario.

India has one of the highest numbers of diabetic population in the world ${ }^{2}$ (topped only by china, recently). A highest 10 year age specific prevalence among $30-79$ years of age ${ }^{3}$ implies that the working class is affected the most. So, the at-risk population in relevance to this study is:

a) Persons between 30 and 70 years of age ${ }^{3}$.

b) Persons who commute to work (i.e., about 70 percent of working population of 487 million) ${ }^{4,5}$ and have a greater change in lifestyle in case of movement restriction.

c) Persons who suffer from diabetes in India (77 million i.e., 7.3 percent crude prevalence.) $)^{6,2,8}$.

d) Pre-diabetic population ${ }^{2}$.

In effect, about 26.9 million peopleare at risk of having deterioration of glycaemic control under movement restriction (cumulative risk assessed by combining the workforce percentage with diabetes prevalence $^{7,2,8}$ ).

\section{Materials \& Methods}

The study was conducted during the National Lockdown(s) with the help of voluntary collection of data by people having diagnosed diabetes. A questionnaire $^{1}$ was disseminated on social media platforms as a Google Documents Form ${ }^{1}$.

Informed consent was obtained from respondents. The participants were required to submit details of their blood sugar levels self-monitored at home, measured fasting at 6-8AM and Random at 3 PM to 8 PM.Total60 participants gave their data. 27 were found to be relevant to the studyand were divided into two groups. Group A (those who provided self-monitored readings) and Group B. (The participants who did not have selfmonitoring devices, and gave data by reporting the symptoms of hyperglycaemia in the questionnaire). Data was gathered from the first week of April to first week of June, 2020. Data was analysed and extent of increase in blood glucose levels was measured.

Selection of the responses relevant to the study was done on following basis:

- The participant is engaged in moderate (or more) physical activity during a normal day. This includes commute to work, exercise, household chores etc.

- The participant is 30 years or older ${ }^{3}$

- The participant has been diagnosed with Diabetes Mellitus.

\section{Results}

Responses received were divided into two groups: Table I

Group A $\left(2 / 3^{\text {rd }}\right.$ of the respondents): Self monitored sugar at home.

Group B $\left(1 / 3^{\text {rd }}\right.$ of the respondents): No selfmonitoring/symptomatic reportage; Empirical group.

Group A: Blood glucose changes (measured fasting at 6-8AM and Random between 3 and 8 P.M. from first week of April to first week of June, 2020). The extent of increase in blood glucose (combined average of rise in blood glucose- fasting and random.) was as follows: 
$<30 \mathrm{mg} / \mathrm{dL}-16.6$ percent participants, $30-50 \mathrm{mg} / \mathrm{dL}-16.6$ percent, $50-90 \mathrm{mg} / \mathrm{dL}-38.8$ percent, $>100 \mathrm{mg} / \mathrm{dL}-27.7$ percent.

Decrease in reported blood sugar levels- None

Group B: (Empirical group)

55percent reported symptoms suggestive of recent increase in blood glucose levels

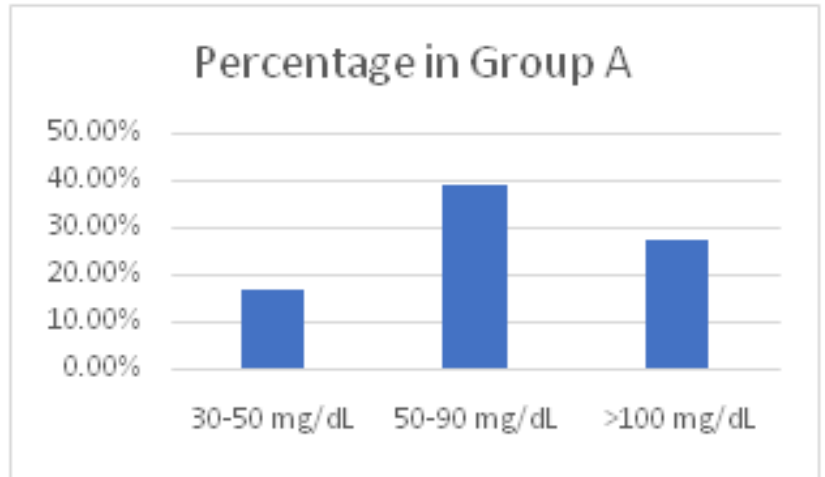

Table I. Percentage of respondents plotted against amount of blood glucose increase.
The findings on the pattern of symptoms experienced (both Group A and Group B) are presented in Table II. These symptoms are associated with hyperglycaemia. It is evident from the data that majority of the respondents (37\%) experienced fatigue/lethargy/tiredness as prominent symptoms. $26.9 \%$ reported either increased thirst or urge to pass urine. Minor skin itching was included in the questionnaire as a screen to differentiate major skin infection from any trivial skin discomfort. 11 percent of respondents did encounter skin infections and had at least $90-100 \mathrm{mg} / \mathrm{dL}$ increase in their fasting blood glucose levels.

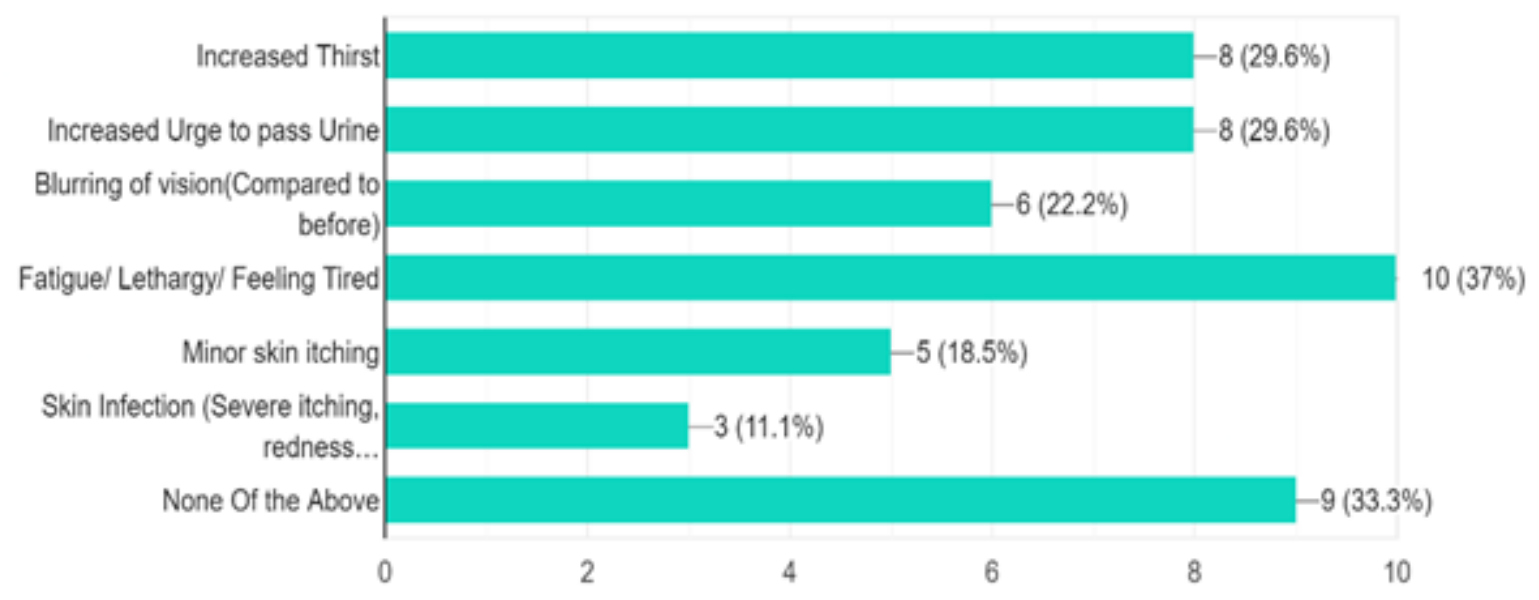

Table II: Symptoms Experienced (Overall in both Group A and B)

\section{Findings about medication and lifestyle changes}

(Figure 1, 2 and 3).77.8 percent of all respondents had been prescribed medication for control of hyperglycaemia (including but not limited to Biguanides and DPP-4 Inhibitors). 19 percent of respondents used insulin (types including but not limited to Humulin 70/30, Glargine, and Detemir). 70percent felt that their lifestyle has significantly changed during lockdown due to movement restriction. 60 percent felt that they are / might be eating larger food portions and only 44 percent had adjusted their food intake vis-à-vis their new daily routine. 59 percent felt that their blood glucose might have increased. 


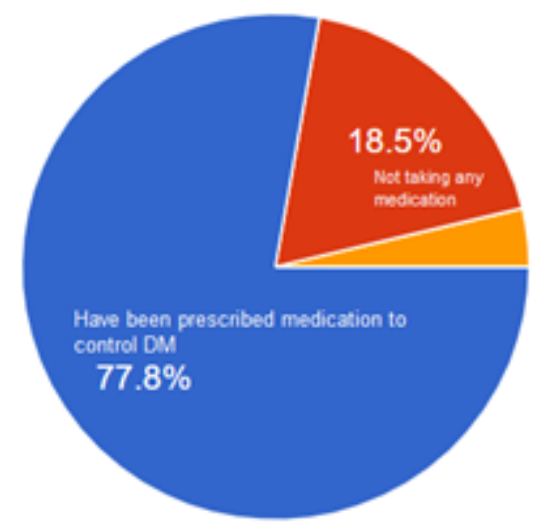

Figure 1: Percentage of participants who take mediation for DM.

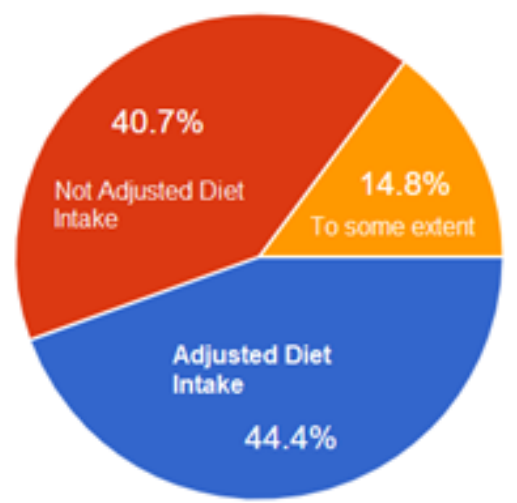

Figure 2: Percentage of participants who have adjusted their food intake with the new daily routine

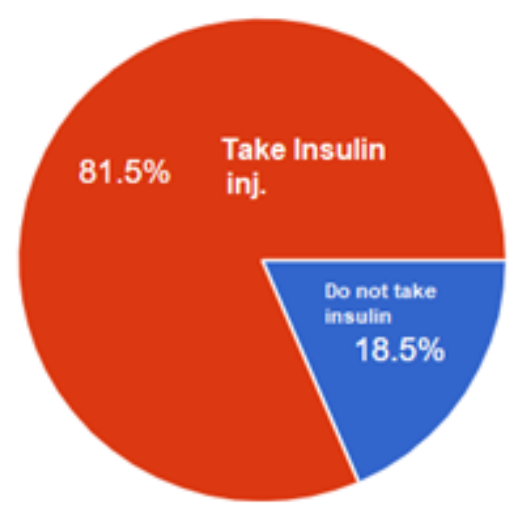

Figure 3: Percentage of participants who take injectable insulin

3 respondents complained of severe skin infection. Upon measuring their blood glucose, they were found to have levels greater than $300 \mathrm{mg} / \mathrm{dL}$ on consecutive occasions. (Dose adjustments and food regulation was advised likewise). This was also the motivation behind conducting this study in the first place.

\section{Results}

This study finds that there is increased incidence of hyperglycaemic episodes in 74 percent of the diabetic population studied. This includes: 55 percent who have documented increase of blood glucose supported by digital monitoring and 19 percent show symptoms of increased blood glucose.

It implies that (of the total 26.9 million at-risk population) 19.9 million people are now likely to have worse glycaemic status (increased blood sugar compared to before) as a result of lockdown. 27.7 percent experienced more than $100 \mathrm{mg} / \mathrm{dL}$ increase in blood glucose compared to before. These people are likely to experience an increase in severity/incidence of complications of "mellitus. Wide spread clinical screening after complete lifting of lockdown is planned to follow up the at-risk population.

At this important juncture, in this time of the coronavirus, there are many people who need to be followed up and safeguarded. They have a very high risk of mortality and complications if they get infected with SARS-CoV-2.Attention and caution in this regard are wanted.

\section{Conclusion}

The change in lifestyle due to lockdowns and restriction of movement thus, affects the diabetic patient.

Clinicians will find it in good stride to look out for correlation between these findings and the complaint that a patient comes to them with. With all regard to previous prescriptions and disease status, dose adjustments and lifestyle adjustments for such patients will be absolutely necessary. Knowledge about effects of movement restriction on the status of Diabetes Mellitus hence, should also be disseminated at the community level, because lifestyle changes as such, can act as potent deterrents. Follow up and research in this area can be planned to further study these effects.

Financial Support and Sponsorship: None Conflicts of Interest: None 


\section{References}

https://doi.org/10.1016/S2213-

1 Google Documents Form: Effect of 8587(17)30174-2.

Lockdown and Movement Restriction on

Glycaemic Status among Diabetics Study https://forms.gle/6xUDsy7N3WkLJU5bA

2 WHO|Country and regional data on diabetes

https://www.who.int/diabetes/facts/world_ figures/en/index $5 . h t m l$

3 The DECODA (Diabetes Epidemiology: Collaborative Analysis of Diagnostic Criteria in Asia) Study Group Department of Epidemiology and Health Promotion, National Public Health Institute, Helsinki, Finland. Age- and Sex-Specific Prevalence of Diabetes and Impaired Glucose Regulation in 11 Asian Cohorts, Diabetes Care 2003 Jun; 26(6): 17701780, https://doi.org/10.2337/diacare.26.6.1770

4 How India commutes to work http://www.indiaenvironmentportal.org.in/ media/iep/infographics/transport/index.ht $\mathrm{ml}$

5 CIA| The World Fact book, South Asia, India

https://www.cia.gov/library/publications/th e-world-factbook/geos/in.html

6 India is home to 77 million diabetics, second highest in the world https://www.thehindu.com/scitech/health/india-has-second-largestnumber-of-people-withdiabetes/article29975027.ece

7 Census of India, Economic Activity https://censusindia.gov.in/census_And_Yo u/economic_activity.aspx

8 Ranjit Mohan Anjana, Mohan Deepa, Rajendra Pradeepa, Jagadish Mahanta Prevalence of diabetes and prediabetes in 15 states of India: results from the ICMRINDIAB population-based cross-sectional study| Lancet Diabetes Endocrinol, $10.1016 /$ S2213-8587(17)30174 